\title{
Offline calibration and monitoring of electromagnetic particle detectors in LHAASO
}

\author{
Hongkui Lv* \\ Key Laboratory of Particle Astrophysics, Institute of High Energy Physics, Chinese Academy of \\ Sciences, Beijing 100049, China \\ E-mail: Ivhkeihep.ac.cn

\section{Yuncheng Nan} \\ Shandong University, Jinan 250100, China \\ Key Laboratory of Particle Astrophysics, Institute of High Energy Physics, Chinese Academy of \\ Sciences, Beijing 100049, China
}

\section{Xiangdong Sheng, Xiaopeng Zhang, Songzhan Chen, Zhe Li, Zheng Wang} Key Laboratory of Particle Astrophysics, Institute of High Energy Physics, Chinese Academy of Sciences, Beijing 100049, China

In the Large High Altitude Air Shower Observatory (LHAASO), one square kilometer array (KM2A), with 5242 electromagnetic particle detectors (EDs) and 1171 muon detectors (MDs), is designed to study ultra-high energy gamma-ray astronomy and cosmic ray physics. The first installed 33 EDs array has been running steadily since February 2018. A set of offline detector self-calibration and data quality monitoring, which relies on the measurement of charged particles within the extensive air showers, has been applied in the data analysis. In this work, the experimental setup and the offline time calibration are described. Finally, observations of the expected moon and sun's shadows of cosmic rays verify the time calibration method.

36th International Cosmic Ray Conference -ICRC2019-

July 24th - August 1st, 2019

Madison, WI, U.S.A.

*This work is supported by the National Key R\&D Program of China (No. 2018YFA0404201) and the Natural Sciences Foundation of China (No. 11605208).

†Speaker. 


\section{Introduction}

LHAASO is a new generation ground-based extensive air shower detector (EAS) array located at the Haizi mountain, China. It is a complex EAS array and consists of three sub-arrays: one squire KiloMetre Array (KM2A), the 78,000 $\mathrm{m}^{2}$ Water Cherenkov Detector Array (WCDA), and the Wide-Field Air Cherenkov Telescope Array (WFCTA), as shown in Fig. 1. The KM2A experiment aims to explore the gamma-ray sources with a sensitivity of $1 \% I_{\text {crab }}$ at energies above $50 \mathrm{TeV}$ $[1,2]$. In its one squire kilometre array (Fig. 1), 5242 electromagnetic particle detectors (EDs) are designed to detect arrival times and number densities of charged particles in the EAS, from which the primary direction and energy can be reconstructed [3], and 1171 muon detectors (MDs) are designed to measure muonic components in the EAS.

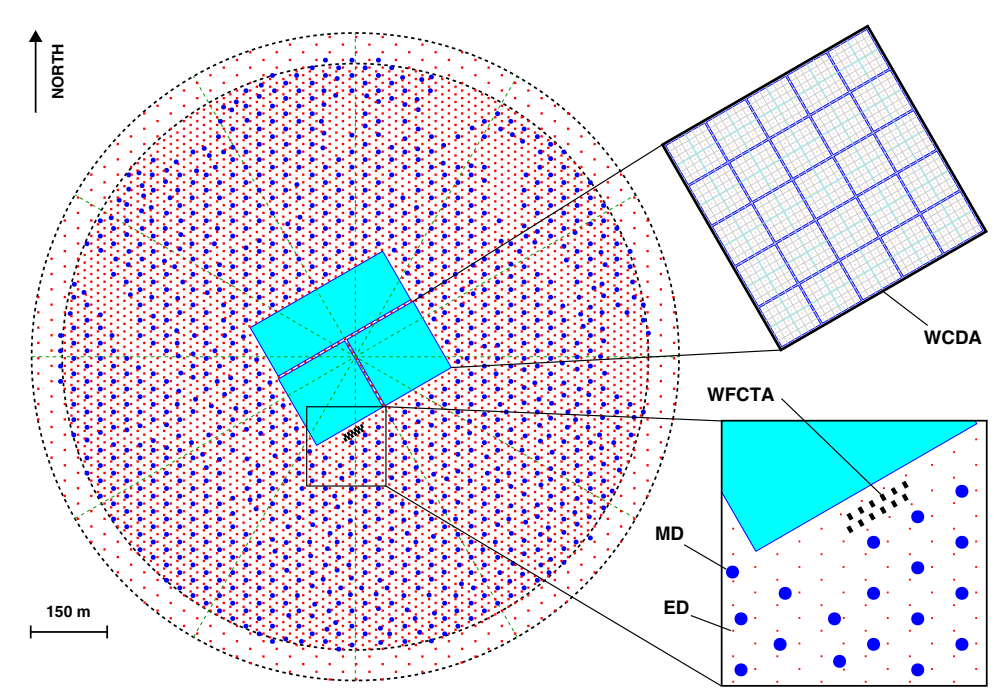

Figure 1: The layout of the LHAASO experiment. LHAASO consists of one square kilometer array (KM2A), water Cherenkov detector array (WCDA) and wide-field Cherenkov telescope array (WFCTA). It covers a total area of approximately $1.2 \mathrm{~km}^{2}$.

An reliable reconstruction of the primary gamma ray direction requires the accurate determination of the arrival times of the EAS particles on each EDs. One of the critical requirements is keeping all the detectors time synchronized within a precision of $1 \mathrm{~ns}$. Hardware time calibration is usually performed using a probe detector manually moved above all the detector units as reference. However, it becomes infeasible if the EAS array has a large area above square kilometer scale and numerous detectors. An automatic detector time self-calibration technique which relies on the measurement of charged particles within the EASs, is implemented in the Monte Carlo simulation and initially applied in 33 EDs array, focusing on its applicability to the upcoming LHAASO-KM2A.

\section{Time calibration}

The ED is a type of scintillation detector with an active area of $1 \mathrm{~m}^{2}$. It consists of four plastic scintillation tiles of $100 \mathrm{~cm} \times 25 \mathrm{~cm} \times 2.5 \mathrm{~cm}$ each, several wavelength-shifting (WLS) fibers and a 1.5 inch photomultiplier tube (PMT), as shown in Figure 2. The ED front-end electronics 
is a very compact device deployed just behind the PMT of each ED [4]. All FEE time-to-digital converters (TDCs) are synchronized within sub-nanosecond via an advanced timing system named White Rabbit [5].
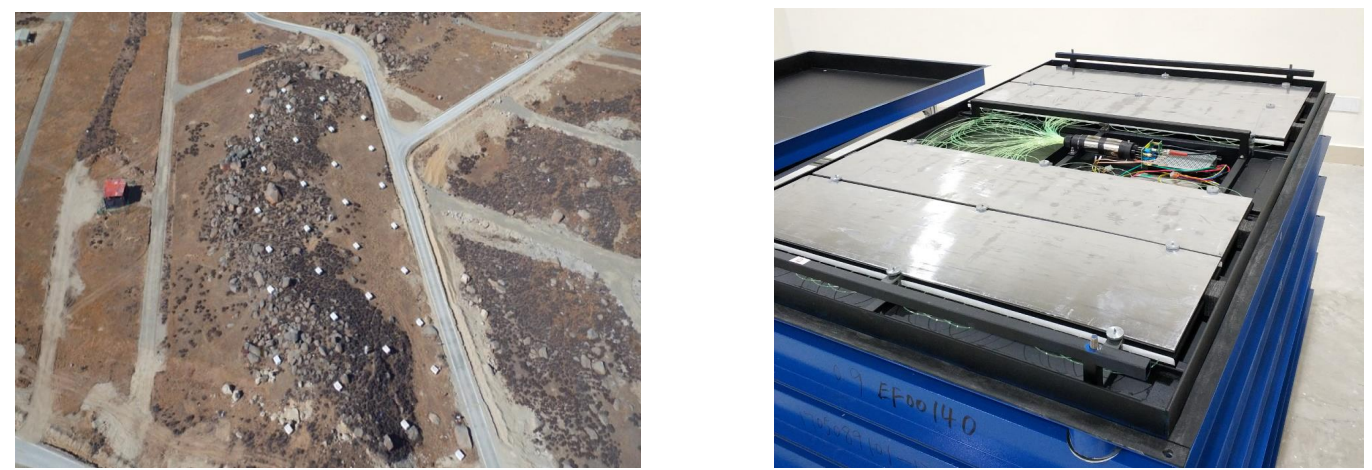

Figure 2: Left figure: The layout of the 33 EDs array in LHAASO experiment; Right figure: Schematic of a ED including 4 scintillation tiles coupled with wavelength-shifting fibers, one PMT and one FEE.

The main uncertainties on the measured arrival time of EAS particles comes from the time offset spread among the EDs. For each ED, the detector time offset arise from the time elapsed between the the incidence of EAS particle arriving on the scintillation tiles and the time stamping of the associated signal in the FEE. It is measured as a cumulative effect of the photon transmission time in the WLS fibers and the electron transit time in the PMTs. The relative time offset differences must be calibrated with a precision within sub-nanosecond and periodically corrected in the data, to guarantee the optimal angular resolution and ensure the pointing accuracy.

\subsection{Calibration principle}

We adopted the characteristic plane (CP) method fully described in [6], and we summarize the main conclusions. This method takes the secondary particles in EAS shower as the calibration beam. The secondary particles within the EAS front can provide a common standard timing signal to calibrate the EDs, since the EAS front approximately sustain a conical shape. The relative arrival time of secondary particles within the EAS front can be accurate determined if the shower front shape and the primary direction are well known. For each EAS event, the time offset of the $i$-th ED $\Delta t_{i}$ located at position coordinates $\left(x_{i}, y_{i}\right)$ is determined as follows:

$$
\Delta t_{i}=t_{i}-t_{i}^{\text {real }}=t_{i}-\left[(l-\bar{l}) \frac{x_{i}}{c}+(m-\bar{m}) \frac{y_{i}}{c}+\sqrt{1-(l-\bar{l})^{2}-(m-\bar{m})^{2}} \frac{z_{i}}{c}+\alpha r_{i}+t_{0}\right]
$$

where $t_{i}$ and $t_{i}^{\text {real }}$ are the measured arrival time of EAS particle for $i$-th ED and the expected "real" one, respectively; $l$ and $m$ are two components of the reconstructed direction vector ${ }^{1}$. The reconstructed direction is corrected using the correction parameters $(\bar{l}, \bar{m})$ of the Characteristic Plane as present in [6], which corresponds to the mean value of the direction cosines for a set of EAS events. $\alpha$ is the conicity coefficient for describing the EAS front, $r_{i}$ is the transverse distance of the $i$-th ED from the shower core, $c$ is the speed of light and $t_{0}$ is a fitting parameter of the direction reconstruction which corresponds to the arrival time of the EAS plane in the coordinates $(0,0,0)$.

\footnotetext{
${ }^{1} 1=\sin \theta \cos \phi, m=\sin \theta \sin \phi(\theta$ and $\phi$ are the reconstructed zenith and azimuth angles, respectively $)$
} 


\subsection{Mente Carlo studies}

To verify the applicability of this method and estimate its precision, a complete timing calibration is performed using simulated showers before the actual experiment. About $10^{6}$ EAS events are generated using the CORSIKA software and used for this calibration. The detector parameters used for simulating ED responses are identical to the experimental measuring values. The preset time offset of each ED, which randomly obtained from a Gaussian distribution, is artificially added into the simulated detector response to distort the detector timing.

Comparing of the measured time offsets with the initial ones yields a direct prediction of the precision of the calibration method. As shown in Figure 3, The differences between the measured time offsets and the initial ones indicates that the precision is within $0.1 \mathrm{~ns}$, which is well within the required precision of KM2A.

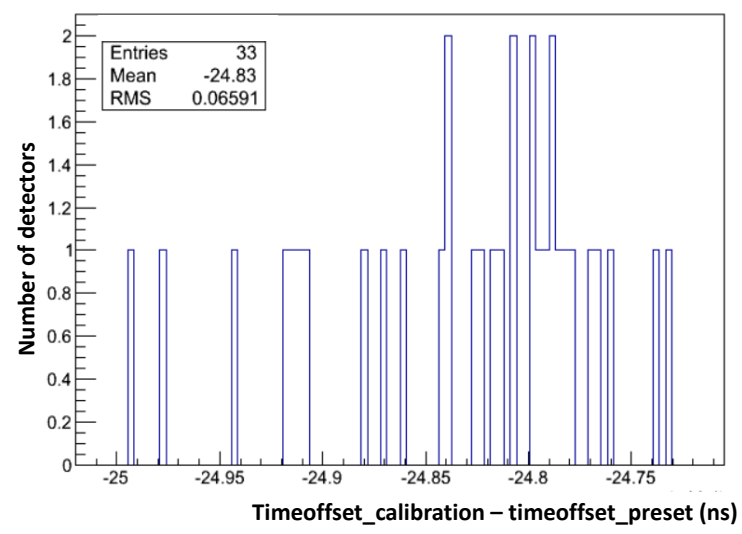

Figure 3: The observed time offsets differences for 33 EDs from their respective preset value.

\subsection{Experimental calibration}

The self-calibration method is implemented in 33 EDs array experiment. The first calibration results have been used in the experiment data over one year to correct the ED time-offset. The quality of direction reconstruction is checked after the ED time offsets are corrected. The reconstructed azimuth angular distribution recovers to a normal distribution after time calibration (Fig. 4), as expected from the simulation.

Finally, the moon's shadow and the sun's shadow are analyzed using 33 EDs array data since February 2018. Cosmic rays are blocked by the moon or the sun and a shadowing effect will be observed on the earth in the directions of the moon or the sun. The analyse of this shadow effect is also efficient checking for the direction reconstruction of EAS array.

As shown in Figure 5, the shadow effect is observed using the data after time calibration. The significance of the moon and sun shadow is $5.9 \sigma$ from $4.4 \sigma$ without calibration. No obvious deviation is observed from the expected positions. The shadowing effects of the moon and the sun agree with expectations, which demonstrate that the $33 \mathrm{EDs}$ is operating as well as expected after time calibration. 


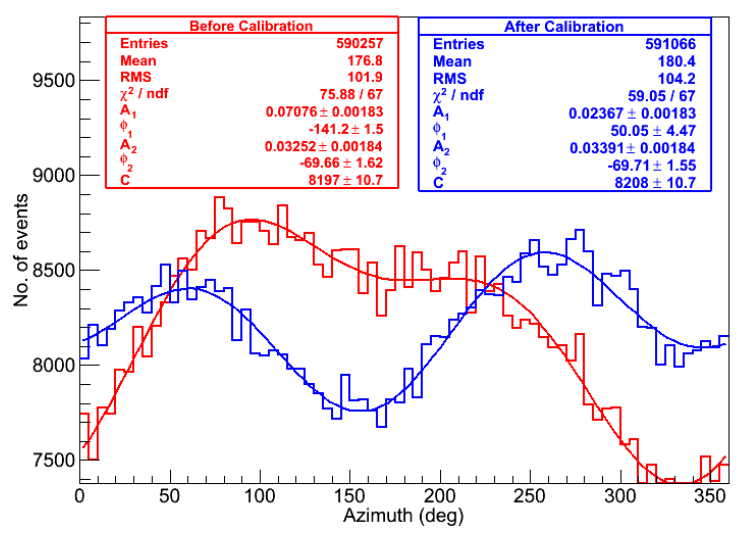

Figure 4: Azimuth distribution before (red line) and after (blue line) the time calibration.
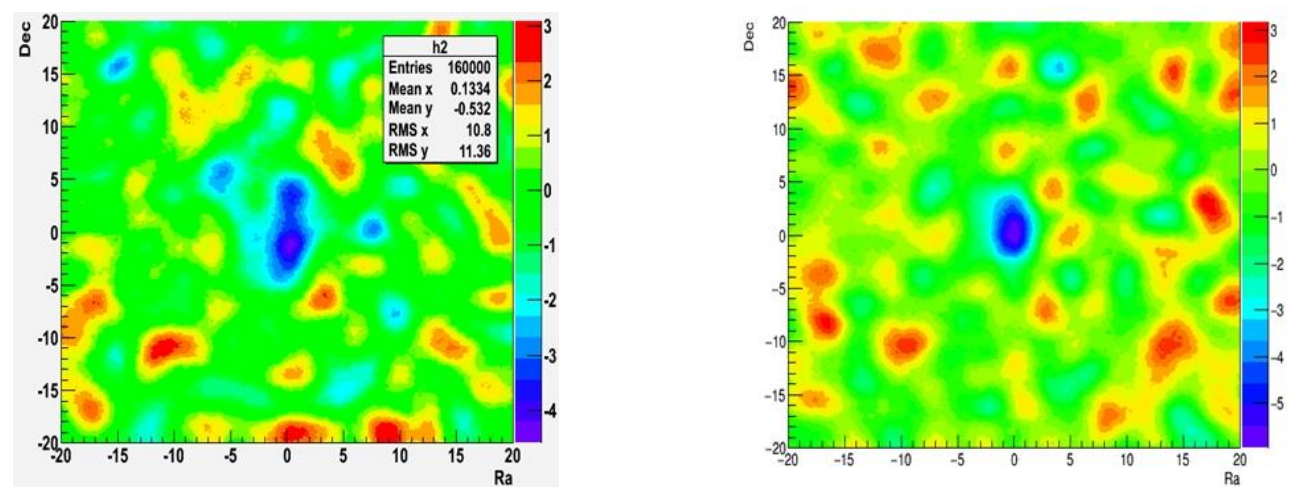

Figure 5: Significance distributions of the moon and sun's shadow without calibration (left) and after calibration (right).

\section{Data quality monitoring}

Until now, 99 EDs and 10 MDs in LHAASO have been installed and running. The first installed 33 EDs have been running steadily for more than one year since February 2018 (Fig. 2). Date quality monitoring is critically important for stable operation of the experiment and the reliability of the recorded data for physics analysis (Fig. 6). A comprehensive monitoring system is developed for this purpose, which comprising detector parameter analysis and automatically checking sub-systems. The system provides filling, archival of monitoring detectors as well as visualization and retrieval of the information to fully meet the needs of operators.

Using this 33 EDs as a platform, the first version of monitoring software is developed. It consists of three steps:

1. Extraction and storage of the relevant information: For each ED, the detector information such as output charge, pedestal are automatically extracted from the off-line data hourly and stored as histogram or scatter diagram (Fig. 7). The detector parameters or summary values are obtained by fitting the histogram and stored in datebase for detector checking.

2. Visualization of the detector parameters: The system compiles the important ED parameters into a map, from which the anomaly detector and its position can be found (Fig. 8). The summary 


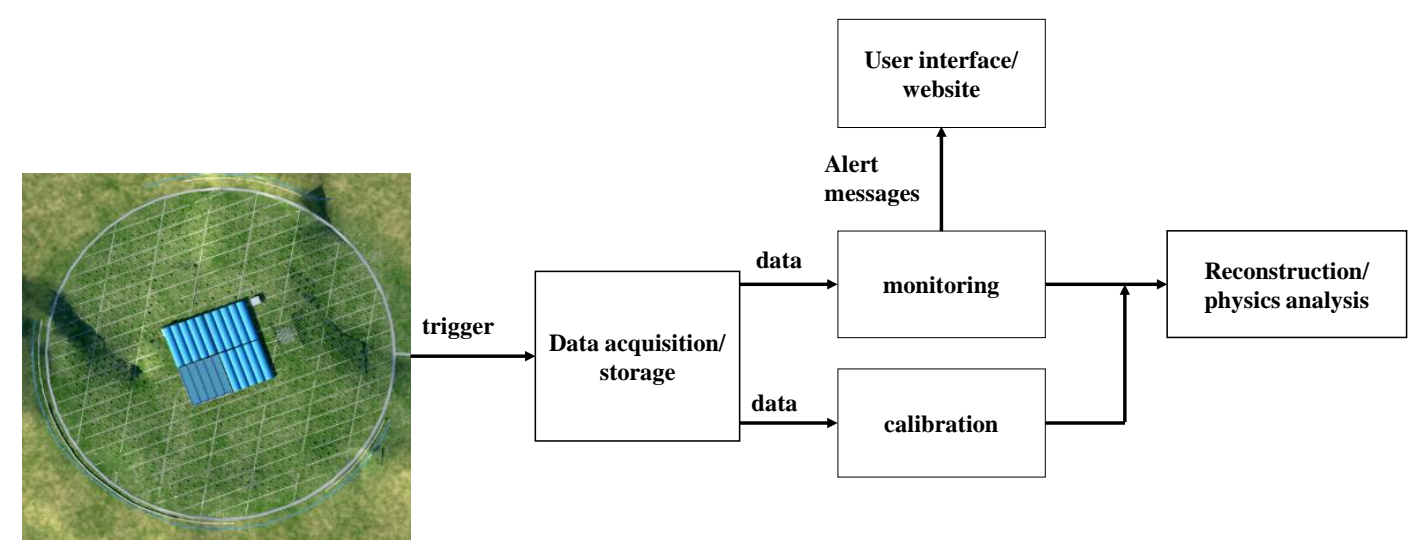

Figure 6: Data flow in the data acquisition system and monitoring system.
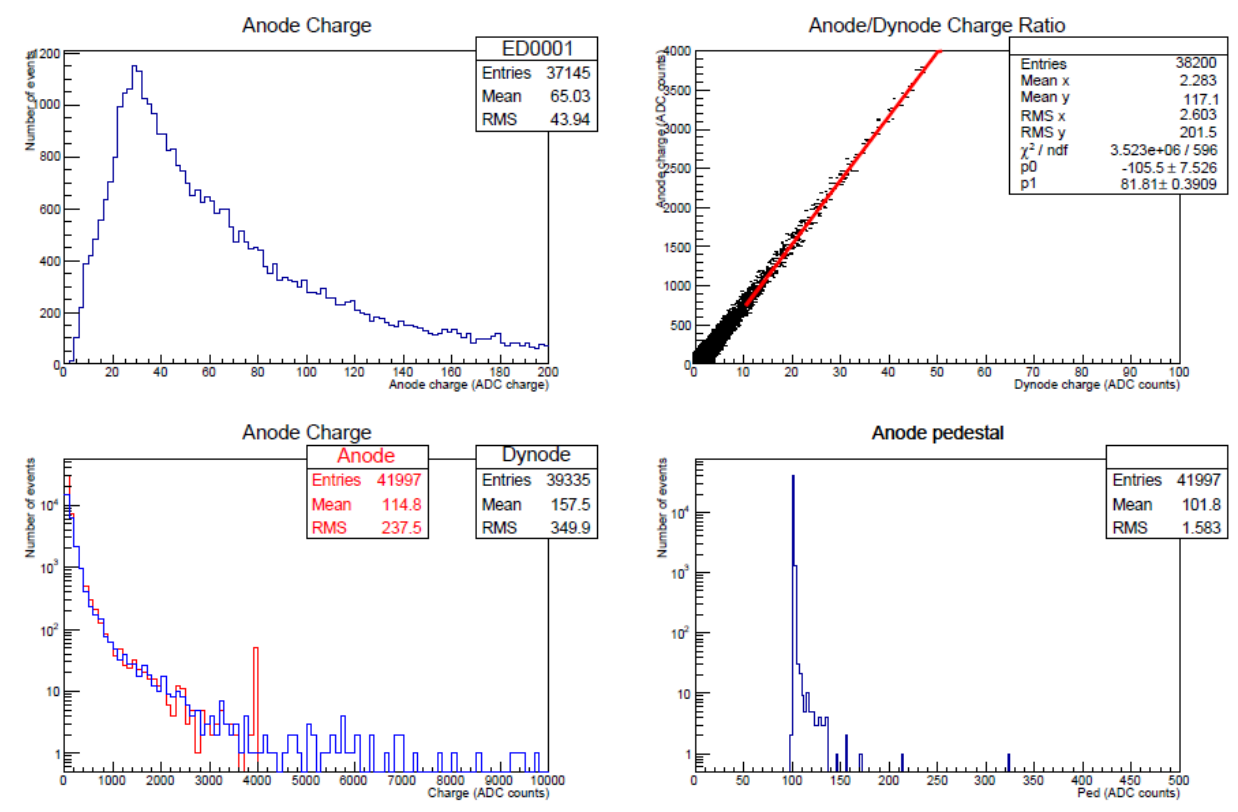

Figure 7: The monitoring diagram of the ED No.1. Upper left: The anode output charge of the ED PMT signal; Upper right: The correlation of the anode charge and dynode charge, from which the gain ratio between anode and dynode is obtained; Bottom left: The anode charge and dynode charge at the same scale; Bottom right: the pedestal of anode signal.

values automatically transport to website on a predefined schedule. The web named "ED data viewer" provides tools for visualization and retrieval of the monitoring elements as trend plots, detector synoptic views, etc (Fig. 9). The ED data viewer can be easily accessed by any user everywhere and intended for prompt feedback.

3. Automatic checking and warning: Exploiting automatic checks by a ROOT based procedure, along with manual checks using ED data viewer, the abnormal detector can be found and warning information will report to the maintenance people. With this tool, system could efficiently identify detector problems. 


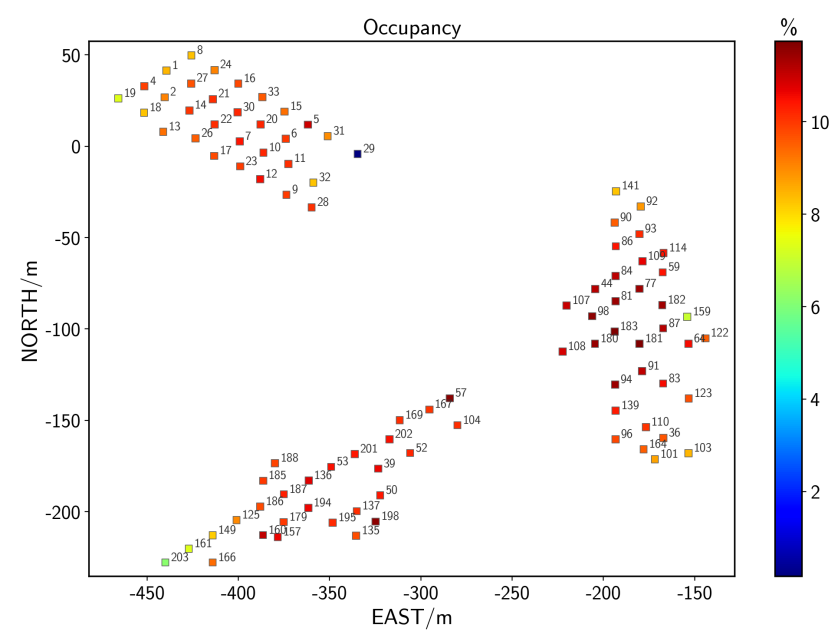

Figure 8: ED map displaying hit occupancy. All the running EDs are represented by the end of June 2019. Color legend indicated the mean hit occupancy per ED.
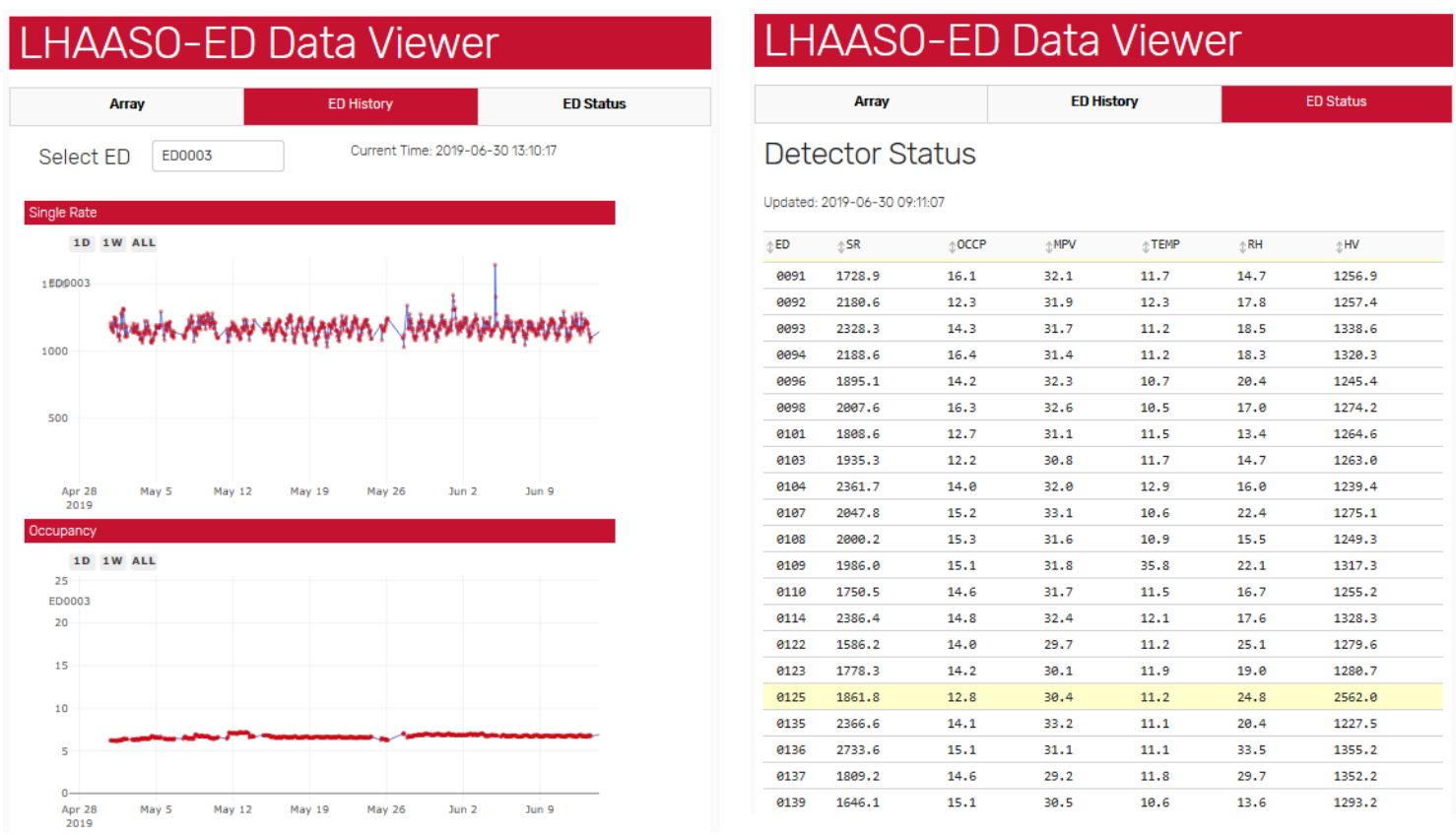

\begin{tabular}{|c|c|c|c|c|c|c|}
\hline †ED & ${ }_{15 R}$ & OCCP & ${ }^{M P V}$ & T TENP & ${ }^{\mathrm{RH}}$ & ${ }_{*}^{H N}$ \\
\hline $8 \bullet 91$ & 1728.9 & 16.1 & 32.1 & 11.7 & 14.7 & 1256.9 \\
\hline 0092 & 2180.6 & 12.3 & 31.9 & 12.3 & 17.8 & 1257.4 \\
\hline 8093 & 2328.3 & 14.3 & 31.7 & 11.2 & 18.5 & 1338.6 \\
\hline 8094 & 2188.6 & 16.4 & 31.4 & 11.2 & 18.3 & 1320.3 \\
\hline 8096 & 1895.1 & 14.2 & 32.3 & 18.7 & 20.4 & 1245.4 \\
\hline 8098 & 2087.6 & 16.3 & 32.6 & 10.5 & 17.8 & 1274.2 \\
\hline${ }^{2101}$ & 1808.6 & 12.7 & 31.1 & 11.5 & 13.4 & 1264.6 \\
\hline 0103 & 1935.3 & 12.2 & 30.8 & 11.7 & 14.7 & 1263.0 \\
\hline 8194 & 2361.7 & 14.8 & 32.8 & 12.9 & 16.8 & 1239.4 \\
\hline 9107 & 2047.8 & 15.2 & 33.1 & 10.6 & 22.4 & 1275.1 \\
\hline 0108 & 2000.2 & 15.3 & 31.6 & 10.9 & 15.5 & 1249.3 \\
\hline 9109 & 1986.8 & 15.1 & 31.8 & 35.8 & 22.1 & 1317.3 \\
\hline e110 & 1758.5 & 14.6 & 31.7 & 11.5 & 16.7 & 1255.2 \\
\hline e114 & 2386.4 & 14.8 & 32.4 & 12.1 & 17.6 & 1328.3 \\
\hline 0122 & 1586.2 & 14.8 & 29.7 & 11.2 & 25.1 & 1279.6 \\
\hline 9123 & 1778.3 & 14.2 & 30.1 & 11.9 & 19.8 & 1280.7 \\
\hline 8125 & 1861.8 & 12.8 & 30.4 & 11.2 & 24.8 & 2562.0 \\
\hline 0135 & 2366.6 & 14.1 & 33.2 & 11.1 & 20.4 & 1227.5 \\
\hline 0136 & 2733.6 & 15.1 & 31.1 & 11.1 & 33.5 & 1355.2 \\
\hline 0137 & 1809.2 & 14.6 & 29.2 & 11.8 & 29.7 & 1352.2 \\
\hline 9139 & 1646.1 & 15.1 & 30.5 & 10.6 & 13.6 & 1293.2 \\
\hline
\end{tabular}

Figure 9: Left: Trend plot of the single channel rate (upper) and occupancy (bottom) versus time with the web ED data viewer. Right: The list of important parameters of monitoring EDs.

\section{Conclusion}

Preliminary result shows that the EAS events is very useful for timing calibration purposes for the large EAS array covering an area for square kilometers scale. Detector time offsets was calibrated using this offline method, which achieve a precision within sub-nanosecond. The observed shadows of the moon and the sun agree with expectations, this results demonstrate the EDs array is operating as well as expected after calibration.

During the first installed EDs data taking since February 2018, the monitoring system demon- 
strated to have a automatics and flexible implementation and has been essential to identify any potential issue as early as possible. This system also improve the understanding of the performance of EDs. The first version of monitoring software is stress-tested at low rate of data taking to verify all algorithms perform as expected. It was possible to set up a more complex algorithm for automatic checking facing the upcoming all EDs running stage.

\section{References}

[1] Zhen Cao, Nucl. Instr. and Meth. A,742(2014),95-98.

[2] Shuwang Cui, et al., Astropart. Phys., 54 (2014) 861́C92 .

[3] Zhongquan Zhang, et al., Nucl. Instr. and Meth. A,845(2017),429-433.

[4] Xiang Liu, et al., Chinese Physics C, 2016, 40(7):076101.

[5] Qiang Du, et al., Nucl. Instr. and Meth. A,732(2013),488-492.

[6] H.H. He, et al., Astroparticle Physics, 27(2007),528-532. 